\title{
Characterization, transport and sorption properties of poly(thiol ester amide) thin-film composite pervaporation membranes
}

\author{
Shu-Hsien Huang ${ }^{\mathrm{a}}$, Wei-Li Lin ${ }^{\mathrm{a}}$, Der-Jang Liaw ${ }^{\mathrm{b}}$, Chi-Lan Li ${ }^{\mathrm{c}}$, Se-Tsung Kao ${ }^{\mathrm{a}, \mathrm{d}}$, \\ Da-Ming Wang ${ }^{\mathrm{a}, \mathrm{e}}$, Kueir-Rarn Lee ${ }^{\mathrm{a}, *}$, Juin-Yih Lai ${ }^{\mathrm{a}}$ \\ a $R E$ D Center for Membrane Technology, Department of Chemical Engineering, Chung Yuan University, Chung-Li 32023, Taiwan \\ ${ }^{\mathrm{b}}$ Department of Chemical Engineering, National Taiwan University of Science and Technology, Taipei 106, Taiwan \\ ${ }^{c}$ Department of Chemical and Material Engineering, Nanya Institute of Technology, Chung-Li 32034, Taiwan \\ ${ }^{\mathrm{d}}$ Institute of Polymer Science and Engineering, National Taiwan University, Taipei 10617, Taiwan \\ e Department of Chemical Engineering, National Taiwan University, Taipei 10617, Taiwan
}

\section{A R T I C L E I N F O}

\section{Article history:}

Received 25 March 2008

Received in revised form 14 May 2008

Accepted 15 May 2008

Available online 27 May 2008

\section{Keywords:}

Pervaporation

Poly(thiol ester amide)

Thin-film composite membrane

Interfacial polymerization

Positron annihilation spectroscopy

Slow positron beam

\begin{abstract}
A B S T R A C T
The effect of acyl chloride chemical structure on the ethanol aqueous solution dehydration through the poly(thiol ester amide) thin-film composite membrane prepared by reacting 2-aminoethanethiol (AETH) with trimesoyl chloride (TMC) or succinyl chloride (SCC) on the surface of the modified asymmetric polyacrylonitrile (mPAN) membrane was investigated. SEM/EDX, ATR-FTIR and water contact angle were applied to analyze the $S$ element, chemical structure, and hydrophilicity of the poly(thiol ester amide) active layer of the composite membrane. In order to estimate the variation in the free volume of the poly(thiol ester amide) active layer and correlate that with the pervaporation performance, positron annihilation spectroscopy (PAS) experiments were conducted, in which a variable monoenergy slow positron beam was used. Doppler broadening $S$ parameters of annihilation radiation energy spectra showed a significant variation with the acyl chloride chemical structures of the poly(thiol ester amide) active layers. The $S$ parameters of the AETH-TMC/mPAN thin-film composite membrane were found to be lower than those of the AETH-SCC/mPAN thin-film composite membrane. In the ethanol aqueous solution dehydration, the AETH-TMC/mPAN thin-film composite membrane exhibited a lower permeation rate and a higher water concentration in the permeate than the AETH-SCC/mPAN. This is in good agreement with the analysis by positron annihilation spectroscopy. The solution effect dominated the pervaporation separation behavior of the poly(thiol ester amide) thin-film composite membrane with TMC substituting for SCC in the poly(thiol ester amide) active layer. The AETH-TMC/mPAN membrane was found to exhibit superior performance compared with some membranes discussed in the literature.
\end{abstract}

(C) 2008 Elsevier B.V. All rights reserved.

\section{Introduction}

Ethanol aqueous solution, a biofuel, comes from the process of fermenting sugar in biomass resources such as the lees and stems of corn. It is one of the new energy sources that would avoid problems concerned with the lack of petroleum resources [1]. Traditional distillation process usually concentrates ethanol aqueous solutions, but the azeotrope restricts further concentration. To obtain ethanol with high purity, excess water needs to be removed using azeotropic distillation with an azeotrope-breaking component (entrainer) or the hybrid system of multi-stage evapo-

\footnotetext{
* Corresponding author at: R\&D Center for Membrane Technology, Department of Chemical Engineering, Chung Yuan University, Chung-Li 32023, Taiwan. Tel.: +886 3 2654190; fax: +88632654198.

E-mail address: krlee@cycu.edu.tw (K.-R. Lee).
}

ration and distillation, which consumes large energy and, therefore, entails cost. Pervaporation, which can separate azeotropic mixtures and save energy and cost, can be a substitute for traditional separation processes, and it is utilized for the dehydration of ethanol aqueous solutions.

Polyamide is an excellent membrane material because of its high thermal stability, excellent mechanical strength, and high resistance to organic solvents. Although dense polyamide membranes show high selectivity in the dehydration of alcohol at wide range of water concentrations, they have low permeation rates [2-4]. In order to promote the permeation rate across polyamide membrane without sacrificing selectivity, the membrane morphology must be converted from a dense thick film into an asymmetrical or a composite morphology. However, the performance of a singlematerial asymmetrical membrane is restricted by its characteristic. A composite membrane would be better as it is characterized by a selective toplayer formed onto a chemically different asymmetri- 
Aminothiol:

$$
\mathrm{AETH}_{\mathrm{SH}}
$$

Acyl chlorides:

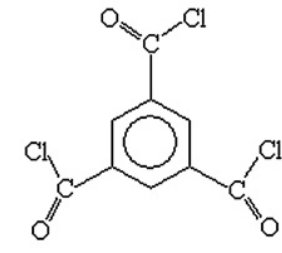

TMC

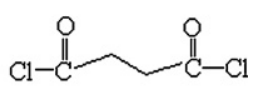

SCC
Fig. 1. Chemical structures of the monomers used in preparing poly(thiol ester amide) active layers.

cal porous membrane substrate. The combination of independent polymeric layers can be advantageous as far as obtaining the desired membrane performance is concerned.

Interfacial polymerization is a useful technique to prepare a composite membrane with a thin selective toplayer. Interfacially polymerized thin-film composite membranes are usually studied in reverse osmosis [5-9] or nanofiltration [10-14], but there have been a few reports on pervaporation [15-17]. Therefore, in this study, two acyl chlorides (trimesoyl chloride (TMC) with three acyl chloride functional groups and succinyl chloride (SCC) with the two acyl chloride functional groups) and the linear aminothiol (2-aminoethanethiol, AETH) were used to prepare the poly(thiol ester amide) active layer on the modified polyacrylonitrile (mPAN) support membrane surface via interfacial polymerization. The effect of the acyl chloride chemical structure on the pervaporation separation behavior of a $90 \mathrm{wt} \%$ ethanol aqueous solution was investigated. To estimate the variation in the free volume of the poly(thiol ester amide) active layer and correlate that with pervaporation performance, the poly(thiol ester amide) thin-film composite membrane was tested by conducting positron annihilation spectroscopy (PAS) experiments, in which a variable monoenergy slow positron beam was used. The reports in regard to this potential physical technique applied to estimate the variation in free volume of thin-film composite pervaporation membranes are few $[18,19]$. The effect of other physicochemical properties, such as hydrophilicity and thickness of the poly(thiol ester amide) active layer, on the pervaporation performance was examined in this study.

\section{Experimental}

\subsection{Materials}

Polyacrylonitrile (PAN) polymer was supplied by Tong-Hua Synthesis Fiber Co. Ltd. in Taiwan. N-Methyl-2-pyrrolidone (NMP) of reagent grade was used as solvent. Trimesoyl chloride and 2-aminoethanethiol were purchased from TCI Co. Succinyl chloride was purchased from Aldrich Co. AETH is an aqueous phase monomer. TMC and SCC are organic phase monomers. Both are used for interfacial polymerization in preparing the poly(thiol ester amide) active layer. Distilled water was used in preparing aminothiol aqueous solution, and reagent-grade toluene was used as the acyl chloride solvent. The chemical structures of monomers used to prepare the poly(thiol ester amide) active layers via interfacial polymerization are shown in Fig. 1.

\subsection{Preparation of modified PAN porous support membrane}

The procedure for the preparation of the modified PAN porous support membrane was taken from our previous study [17]. An NMP solution containing $15 \mathrm{wt} \%$ PAN polymers was cast onto a polyester nonwoven substrate with a casting knife with $200-\mu \mathrm{m}$ gap to prepare the flat porous PAN support. The cast membrane was precipitated in a water bath. The resulting PAN porous support membrane was washed in water several times for more than 1 day to remove the remnant of NMP solvent. The modified PAN porous membrane was prepared by immersing the PAN support in a $2 \mathrm{M}$ $\mathrm{NaOH}$ solution at $50^{\circ} \mathrm{C}$. The partial $-\mathrm{CN}$ groups of PAN can be converted into $-\mathrm{COOH}$ groups after the hydrolysis with $\mathrm{NaOH}$ solution [10]. The mPAN porous support membrane was washed in a water bath.

\subsection{Preparation of poly(thiol ester amide) thin-film composite membrane}

The poly(thiol ester amide) thin-film composite membrane was prepared via the interfacial polymerization of aminothiol (AETH) and acyl chloride (TMC or SCC). The mPAN membrane was first immersed in a $0.01 \mathrm{wt} \%$ aminothiol aqueous solution for $10 \mathrm{~s}$. Then, the excess amount of aminothiol aqueous solution on the surface of the mPAN membrane was removed. The mPAN membrane soaked in the aminothiol aqueous solution was contacted with a $0.05 \mathrm{wt} \%$ acyl chloride organic solution for $10 \mathrm{~s}$ to carry out interfacial polymerization. Finally, the resulting poly(thiol ester amide) thin-film composite membrane was washed in methanol and then dried at atmospheric temperature.

\subsection{Characterization}

ATR-FTIR(PerkinElmer Spectrum One) spectroscopy was used to characterize the chemical structure of the active layer of the thinfilm composite membrane. The surface $S$ element distribution of the poly(thiol ester amide) composite membrane was monitored using SEM/EDX (HITACHI S-3000N). In order to understand the surface hydrophilicity of the poly(thiol ester amide) thin-film composite membrane, the water contact angle was estimated with the use of Automatic Interfacial Tensiometer (FACE Mode 1 PD-VP). To estimate the variations in the free volume in the multilayer structure of the poly(thiol ester amide) thin-film composite membrane, a variable monoenergy slow positron beam as a function of positron incident energy $(0-30 \mathrm{keV})$ at atmospheric temperature under a vacuum of $\sim 10^{-8}$ Pa was used to obtain Doppler broadening energy spectrum (DBES). Doppler-broadened linewidth can be described by using simple lineshape parameters, such as an $S$ (shape) parameter. The $S$ parameter defined by the ratio of the central part of the annihilation spectrum to the total spectrum reflects positron annihilation with low momentum valence electrons. To estimate the thickness of the poly(thiol ester amide) active layer, the $S$ parameter data from DBES was fitted in a four-layer model by VEPFIT program.

\subsection{Pervaporation measurement}

The pervaporation separation of ethanol aqueous solution through the poly(thiol ester amide) thin-film composite membrane was performed. The pervaporation apparatus was presented in our previous study [20]. The feed solution was contacted with the membrane directly. The effective area of the membrane for pervaporation was $11.64 \mathrm{~cm}^{2}$. The operating temperature (feed solution temperature) was $25^{\circ} \mathrm{C}$. The concentrations of the feed solution and permeate were measured by gas chromatography (GC; China 
(a)

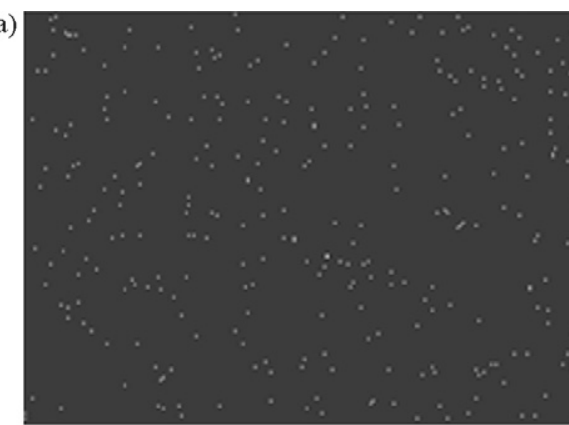

S Kal

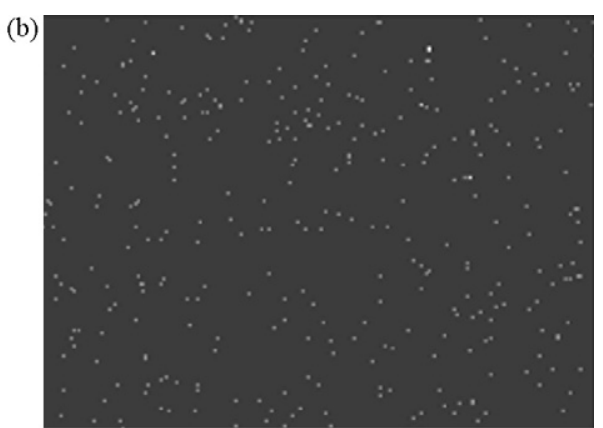

$\mathrm{S} \mathrm{Kal}$

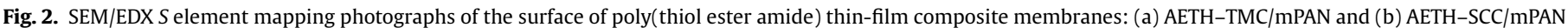
$(100 \times)$.

Chromatography $8700 \mathrm{~T})$. The permeation rate $(P)$ and pervaporation separation factor of water/ethanol $\left(\alpha_{\mathrm{P}}\right)$ were calculated from the following equations:

$P=\frac{W}{A t}$

$\alpha_{\mathrm{P}}=\frac{Y_{\mathrm{W}} / Y_{\mathrm{E}}}{X_{\mathrm{W}} / X_{\mathrm{E}}}$

where $W$ is the weight of the permeate, $A$ is the effective membrane area, $t$ is the sampling time, $X_{\mathrm{W}}$ and $X_{\mathrm{E}}$ are the respective weight fractions of water and ethanol in the feed. $Y_{\mathrm{W}}$ and $Y_{\mathrm{E}}$ are the respective weight fractions of water and ethanol in the permeate.

\subsection{Sorption measurement}

The sorption measurement and apparatus was presented in our previous study [21]. A specially designed device was used in this experiment, which allows only the poly(thiol ester amide) side of the composite membrane contact the feed solution at $25^{\circ} \mathrm{C}$. The poly(thiol ester amide) side of the membrane was subsequently blotted with a filter paper to remove the excess solution, and the membrane was instantly placed in the left container of a twintube setup to be frozen by the liquid nitrogen. The system was evacuated with the use of a vacuum pump. The left container holding the swollen membrane was heated to evaporate completely the liquid absorbed in the membrane. The evaporated vapor was trapped in the right container, which is cooled with liquid nitrogen. The composition of the condensed liquid in the right container was measured with a GC. The solution separation factor of water/ethanol $\left(\alpha_{\mathrm{S}}\right)$ was calculated from the following equation:

$\alpha_{\mathrm{S}}=\frac{Z_{\mathrm{W}} / Z_{\mathrm{E}}}{X_{\mathrm{W}} / X_{\mathrm{E}}}$

where $X_{\mathrm{W}}$ and $X_{\mathrm{E}}$ are the respective weight fractions of water and ethanol in the feed. $Z_{\mathrm{W}}$ and $Z_{\mathrm{E}}$ are the respective weight fractions of water and ethanol in the membrane.

\section{Results and discussion}

\subsection{Characterization of poly(thiol ester amide) thin-film composite membrane}

In order to characterize the poly(thiol ester amide) polymerized on the surface of the MPAN, SEM/EDX and ATR-FTIR were used. SEM/EDX measured the $S$ element, and ATR-FTIR measured the chemical structure. Fig. 2 shows the SEM/EDX $S$ element mapping photographs of the surface of the poly(thiol ester amide) thinfilm composite membranes. They reveal that $S$ elements (bright

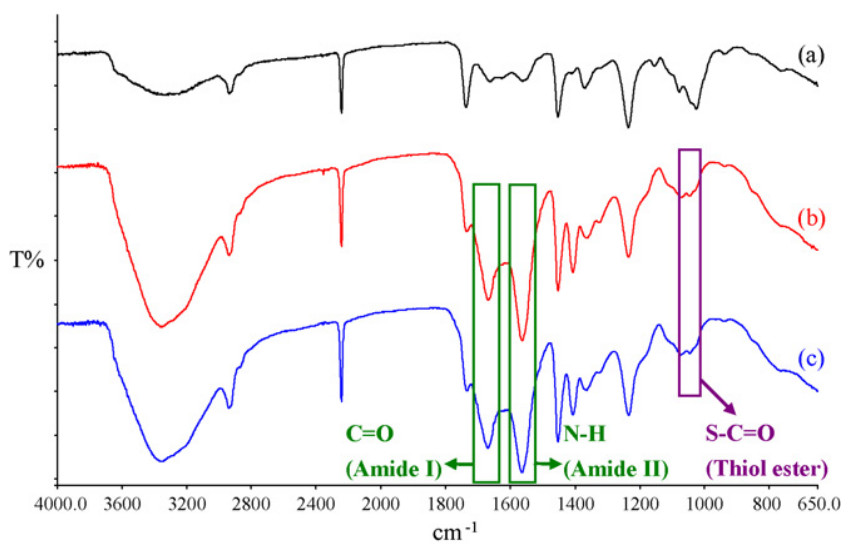

Fig. 3. ATR-FTIR spectra of (a) modified PAN membrane and poly(thiol ester amide) composite membranes, (b) AETH-TMC/mPAN and (c) AETH-SCC/mPAN.

dots) are evenly spread over the surface of AETH-TMC/mPAN and AETH-SCC/mPAN thin-film composite membranes. The ATR-FTIR spectra of the mPAN and poly(thiol ester amide) thin-film composite membranes are exhibited in Fig. 3. Compared with the spectrum of mPAN (Fig. 3(a)), the spectra of AETH-TMC/mPAN and AETH-SCC/mPAN membranes shown in Fig. 3(b) and (c) indicate three peaks, which correspond to $\mathrm{C}=\mathrm{O}$ (amide $\mathrm{I}$ ), $\mathrm{N}-\mathrm{H}$ (amide II) and $\mathrm{S}-\mathrm{C}=\mathrm{O}$ (thiol ester) at wave numbers 1667, 1563 and $1044 \mathrm{~cm}^{-1}$, respectively. The above-mentioned results of ATR-FTIR and SEM/EDX confirmed that the active layers of the thin-film composite membranes are composed of poly(thiol ester amide)s.

\subsection{Effects of acyl chloride chemical structure and feed composition on pervaporation performance}

Poly(thiol ester amide)s prepared with AETH and acyl chlorides with different chemical structures had different performance for the pervaporation separation processes. The effect of acyl chloride chemical structure on the pervaporation separation of a $90 \mathrm{wt} \%$ ethanol aqueous solution at $25^{\circ} \mathrm{C}$ was studied, and the resulting data is exhibited in Table 1. The AETH-TMC/mPAN thin-film

Table 1

Effect of acyl chloride structure on pervaporation performance of $90 \mathrm{wt} \%$ aqueous ethanol solution through poly(thiol ester amide) thin-film composite membranes at $25^{\circ} \mathrm{C}$

\begin{tabular}{lll}
\hline Membranes & $\begin{array}{l}\text { Water concentration } \\
\text { in permeate }(\text { wt } \%)\end{array}$ & $\begin{array}{l}\text { Permeation rate } \\
\left(\mathrm{g} /\left(\mathrm{m}^{2} \mathrm{~h}\right)\right)\end{array}$ \\
\hline AETH-TMC/mPAN & $99.2 \pm 0.2$ & $1586 \pm 120$ \\
$\mathrm{AETH}-\mathrm{SCC} / \mathrm{mPAN}$ & $92.7 \pm 1.4$ & $1749 \pm 239$ \\
\hline
\end{tabular}


Table 2

Effect of acyl chloride structure on sorption and diffusion properties of poly(thiol ester amide) thin-film composite membranes for $90 \mathrm{wt} \%$ aqueous ethanol solution at $25^{\circ} \mathrm{C}$

\begin{tabular}{lccl}
\hline Membranes & $\alpha_{\mathrm{P}}$ & $\alpha_{\mathrm{S}}$ & $\alpha_{\mathrm{D}}$ \\
\hline AETH-TMC/mPAN & $1129.6 \pm 284.9$ & $107.5 \pm 29.3$ & $10.8 \pm 1.3$ \\
AETH-SCC/mPAN & $110.6 \pm 29.2$ & $7.2 \pm 1.2$ & $15.2 \pm 1.8$ \\
\hline
\end{tabular}

$\alpha_{\mathrm{P}}=$ pervaporation separation factor; $\alpha_{\mathrm{S}}=$ solution separation factor; $\alpha_{\mathrm{D}}=$ diffusion separation factor.

composite membrane was found to show lower permeation rate and higher water concentration in the permeate compared to the AETH-SCC/mPAN thin-film composite membrane. This may be because the aromatic TMC has a rigid phenyl ring and three acyl chloride groups. The crosslinking network structure could be formed in the AETH-TMC active layer from reacting AETH with TMC, resulting in the more stable poly(thiol ester amide) active layer on the AETH-TMC/mPAN thin-film composite membrane and the restriction on the swelling effect of the ethanol-water feed solution. This resulted in a higher water concentration in the permeate and a lower permeation rate. On the other hand, the aliphatic SCC has a linear structure and two acyl chloride groups. When SCC reacted with $\mathrm{AETH}$, the crosslinking structure could not be formed in the poly(thiol ester amide) active layer of AETH-SCC/mPAN thin-film composite membrane. As a result, AETH-SCC could be swollen easily by the ethanol-water feed solution. Because of excessive swelling, the selective component $\left(\mathrm{H}_{2} \mathrm{O}\right)$ carried the nonselective component (EtOH) through the AETH-SCC/mPAN thin-film composite membrane and to the permeate. Thus, an opposite pervaporation separation trend was obtained in comparison to the AETH-TMC/mPAN thin-film composite membrane.

Based on the solution-diffusion model, the permeability coefficient is determined by multiplying the solution coefficient with the diffusion coefficient. Similarly, the pervaporation separation factor $\left(\alpha_{\mathrm{P}}\right)$ can be described by the solution separation factor $\left(\alpha_{\mathrm{S}}\right)$ multiplied by the diffusion separation factor $\left(\alpha_{D}\right)$, that is, $\alpha_{P}=\alpha_{S} \alpha_{D}$, where $\alpha_{\mathrm{S}}$ is obtained from the sorption experiments and $\alpha_{\mathrm{D}}$ is calculated from the ratio of $\alpha_{\mathrm{P}}$ to $\alpha_{\mathrm{S}}$. The effect of the acyl chloride structure on the sorption and diffusion properties of the poly(thiol ester amide) thin-film composite membranes for a $90 \mathrm{wt} \%$ ethanol aqueous solutions at $25^{\circ} \mathrm{C}$ was studied, and the resulting data is exhibited in Table 2.

As shown in Table 2, both $\alpha_{\mathrm{P}}$ and $\alpha_{\mathrm{S}}$ for the AETH-TMC/mPAN thin-film composite membrane are higher compared to the AETH-SCC/mPAN thin-film composite membrane. Furthermore, $\alpha_{\mathrm{S}}$ is much higher than $\alpha_{\mathrm{D}}$ for a $90 \mathrm{wt} \%$ ethanol aqueous solution through the AETH-TMC/mPAN thin-film composite membrane at $25^{\circ} \mathrm{C}$. This indicates that the solution effect primarily controls the separation behavior of the pervaporation through the AETH-TMC/mPAN thin-film composite membrane.

The poly(thiol ester amide) prepared with the aliphatic SCC has a linear structure and is easily swollen by the ethanol-water feed solution. This results in an increase in the free volume, which makes the ethanol penetrate into AETH-SCC active layer easily. This causes the lower $\alpha_{\mathrm{S}}$ for the AETH-SCC/mPAN membrane. The poly(thiol ester amide) prepared with the aromatic TMC has a crosslinking network structure, which can restrict the swelling effect of the ethanol-water feed solution. The free volume resulting from the swelling effect of the ethanol-water feed solution is not large enough. Thus, the ethanol molecules cannot be adsorbed easily onto AETH-TMC active layer, whereas the water molecules can preferentially be adsorbed onto AETH-TMC active layer. This results in higher $\alpha_{\mathrm{S}}$ for the AETH-TMC/mPAN thin-film composite membrane.

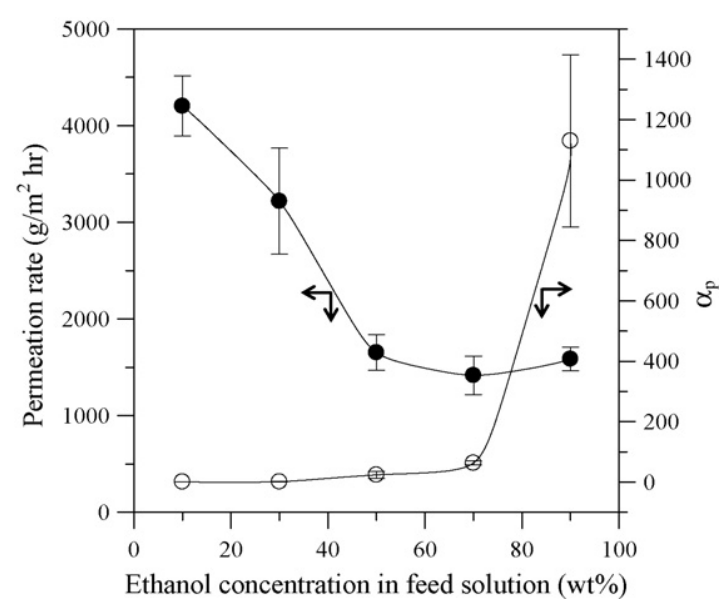

Fig. 4. Effect of ethanol concentration in feed solution on pervaporation performance of AETH-TMC/mPAN composite membrane at $25^{\circ} \mathrm{C}$.

Normally, pervaporation performance strongly depends on feed composition. Pervaporation performance at different feed compositions was investigated. Fig. 4 shows the effect of the ethanol concentration in the feed solution on the pervaporation performance of the AETH-TMC/mPAN composite membrane at $25^{\circ} \mathrm{C}$. The permeation rate was found to increase, but $\alpha_{\mathrm{P}}$ decreased when the ethanol concentration in the feed solution decreased. The AETH-TMC/mPAN composite membrane was a water-preferential membrane, resulting from the hydrophilic AETH-TMC active layer. When the water concentration in the feed solution increased (the ethanol concentration in the feed solution decreased), the driving force, swelling effect and coupling effect increased, which in turn caused an increase in the permeation rate but a decrease in $\alpha_{\mathrm{p}}$. Thus, the AETH-TMC/mPAN composite membrane is favorably applied in the pervaporation separation of a highly concentrated ethanol aqueous solution.

In order to further realize the effect of the acyl chloride structure on the pervaporation performance, physicochemical properties (such as free volume, layer thickness, and hydrophilicity) of the poly(thiol ester amide) active layers prepared with AETH and various acyl chlorides were explored in detail. The details are given in the following sections.

\subsection{Effects of free volume, thickness and hydrophilicity of poly(thiol ester amide) active layer on pervaporation performance}

PAS experiment with a variable monoenergy slow positron beam was performed to obtain DBES. In general, DBES can be described by a lineshape $S$ parameter. The $S$ parameter value increases with increasing the relative contribution of low momentum electrons to the positron annihilation in the open volume defects $[18,19,22]$. DBES can, therefore, be applied to measure the variation in free volume of a composite membrane. The multilayer structure of a composite membrane can be analyzed by the $S$ parameter variation (free volume variation). The data of $S$ parameter vs. positron incident energy (or mean depth) in the AETH-TMC/mPAN and AETH-SCC/mPAN thin-film composite membranes is exhibited in Fig. 5. There are several observations: (1) The $S$ value close to the surface increases steeply with an increase in the positron incident energy. (2) The $S$ value exhibits a short plateau, which deepens some distance from the membrane surface. (3) The $S$ value leaps to another plateau. (4) $S$ increases to a maximum value with an increase in the positron incident energy (mean depth). (5) After the maximum value, $S$ decreases with further increase in the positron incident energy (mean depth). 


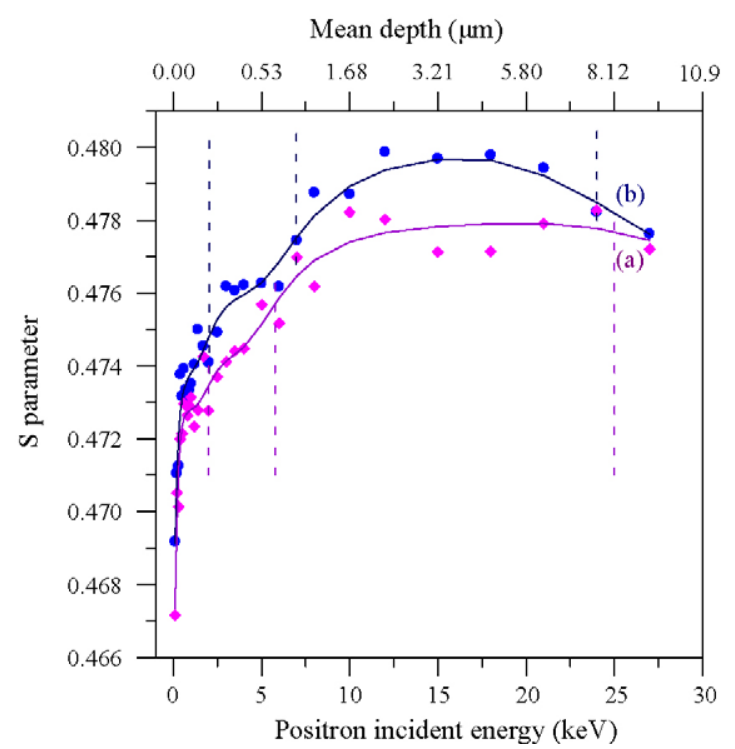

Fig. 5. $S$ parameter vs. positron incident energy (or depth) data for poly(thiol ester amide) thin-film composite membranes prepared with AETH and different acyl chlorides. The lines for $S$ parameters are fitted results in a four-layer model from VEPFIT program analysis. Vertical broken lines indicate boundaries from layer to layer. ( a) AETH-TMC/mPAN and $(\bullet$, b) AETH-SCC/mPAN.

The steep increase in the $S$ value close to the surface is a typical phenomenon of positronium annihilation. The $S$ value exhibits two plateaus; it increases and then decreases with an increase in the positron incident energy $(0.5-27 \mathrm{keV})$. These $S$ parameter variations resulting from variations in free volume in the physical structures reveal a multilayer structure in the membrane from positron annihilation characteristic difference among those layers.

VEPFIT program analysis was applied to fit $S$ data in a four-layer model. The fitted lines shown in Fig. 5 exhibit good fitting results of VEPFIT program analysis for the poly(thiol ester amide) thin-film composite membranes. From the result of VEPFIT program analysis, the poly(thiol ester amide) thin-film composite membrane is divided into four layers, the boundaries of which are indicated by three vertical broken lines shown in Fig. 5 for each composite membrane. For the AETH-TMC/mPAN thin-film composite membrane, for example, three vertical broken lines indicate the boundaries of the four layers. The order of the multilayer structure is as follows: The first two plateaus pertain to the AETH-TMC active layer $(0.5-2.0 \mathrm{keV})$ and the dense skin layer of mPAN (2.0-5.8 keV). The next increase in the $S$ value represents a transition layer from the dense skin layer to the porous support layer of mPAN (5.8-25 keV). Finally, the decrease in the $S$ value represents the porous support layer of mPAN ( $>25 \mathrm{keV})$. The obtained layer structure is schematically shown in Fig. 6. As shown in Fig. 5, the first plateau (the active layer) of the AETH-SCC/mPAN thin-film composite membrane is higher than that of the AETH-TMC/mPAN thin-film composite membrane. This implied that the free volume of the former membrane was higher than that of the latter membrane.

The effective selective layer of the thin-film composite membrane is the poly(thiol ester amide) active layer. Table 3 exhibits the $S$ values corresponding to the free volume and the thickness of the poly(thiol ester amide) active layers estimated by VEPFIT program analysis. Based on the data in Table 3, the $S$ value (free volume) and the thickness of AETH-TMC active layer are smaller than those of AETH-SCC. These phenomena may be due to the crosslinking structure of AETH-TMC active layer prepared with TMC, which has three-functional aromatic groups. This caused the lower free volume. Whereas, AETH-SCC active layer prepared with
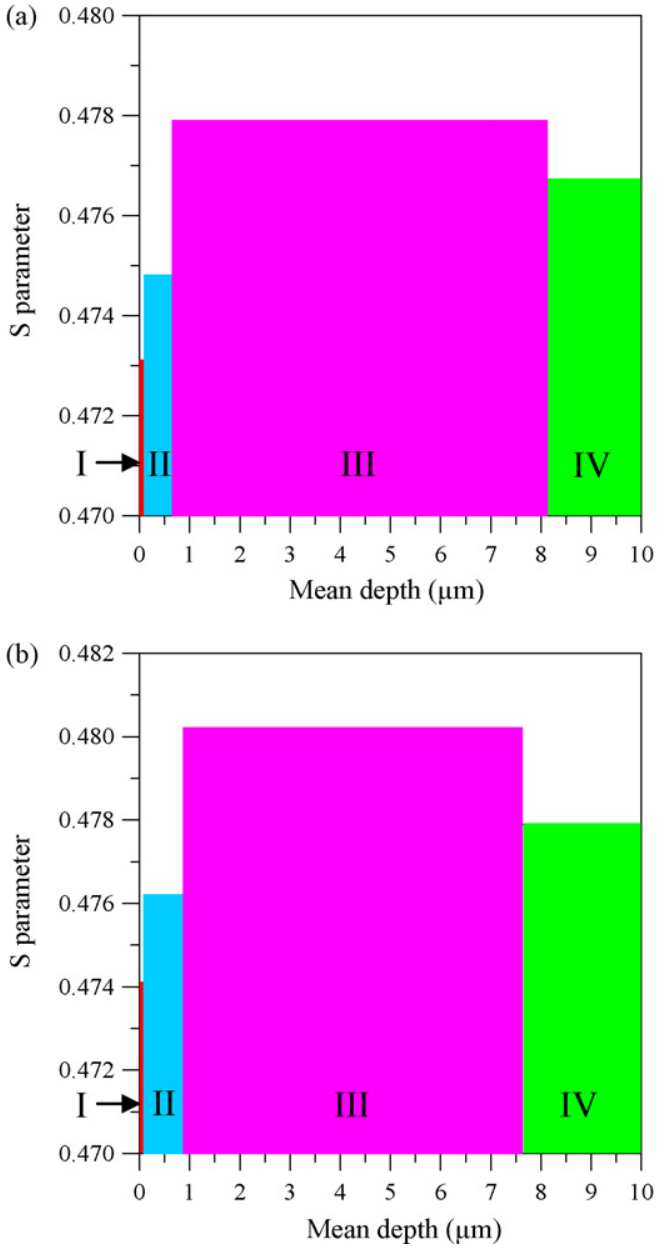

Fig. 6. Schematic diagram of four-layer depth structure obtained by using VEPFIT program analysis of $S$ parameter from DBES in TETA-TMC/mPAN composite membrane. (I) polyamide thin layer, (II) dense skin layer of mPAN, (III) transition layer from dense skin layer to porous support layer of mPAN and (IV) porous support layer of mPAN. (a) AETH-TMC/mPAN and (b) AETH-SCC/mPAN.

SCC, which has two-functional aliphatic groups, has a linear and no crosslinking structure. This caused the higher free volume. Thus, the AETH-TMC/mPAN membrane shows a lower permeation rate and a higher water concentration in the permeate compared to the AETH-SCC/mPAN based from the pervaporation separation of the ethanol aqueous solution. These observations agree very well with the result shown in Table 1 .

Besides the free volume and thickness of the poly(thiol ester amide) active layers, there should be other physicochemical properties, such as the affinity between the feed solution and the poly(thiol ester amide) active layer, to be explored for the pervaporation performance. The membrane material's characteristic is an important key that affects the capability of water to adsorb onto the membrane or the affinity between the feed solution and the

Table 3

$S$ value and thickness of poly(thiol ester amide) active layer of poly(thiol ester amide) thin-film composite membranes prepared with AETH and different acyl chlorides from multilayer analysis of $S$ data in a four-layer model using VEPFIT program

\begin{tabular}{llc}
\hline Poly(thiol ester amide)s & $S$ value $^{\mathrm{a}}$ & Thickness $^{\mathrm{a}}(\mathrm{nm})$ \\
\hline AETH-TMC & $0.4731 \pm 0.0005$ & $101.7 \pm 47.3$ \\
AETH-SCC & $0.4739 \pm 0.0018$ & $103.5 \pm 41.3$
\end{tabular}

a $S$ value and thickness of the first layer from fitted $S$ data by VEPFIT program analysis. 
Table 4

Water contact angle of poly(thiol ester amide) thin-film composite membranes

\begin{tabular}{lc}
\hline Membranes & Water contact angle $\left(^{\circ}\right)$ \\
\hline AETH-TMC/mPAN & $57.9 \pm 2.8$ \\
AETH-SCC/mPAN & $43.3 \pm 2.4$ \\
\hline
\end{tabular}

membrane surface for the dehydration of the water/alcohol mixtures through the pervaporation membrane. The capability of water to wet the membrane surface, that is, the affinity between the water and the membrane surface can be directly estimated using the water contact angle. The lower the water contact angle, the higher is the hydrophilicity, that is to say, the greater is the capability of water to wet the membrane surface. Therefore, the effect of the hydrophilicity of the poly(thiol ester amide) active layers prepared with AETH and acyl chlorides with different chemical structures on the pervaporation separation for the poly(thiol ester amide) thin-film composite membranes was investigated.

The water contact angle of the poly(thiol ester amide) thin-film composite membranes is shown in Table 4. It is indicated that the AETH-TMC/mPAN membrane exhibits a higher water contact angle than the AETH-SCC/mPAN membrane. This phenomenon may be attributed to the poly(thiol ester amide) AETH-TMC active layer prepared with TMC, which has three-functional aromatic groups and a hydrophobic phenyl ring, and to the crosslinking network structure formed during interfacial polymerization. The introduction of hydrophobic phenyl ring in the AETH-TMC active layer resulted in a higher water contact angle than the AETH-SCC active layer. The AETH-TMC active layer was swollen by the ethanol in the feed solution more easily than the AETH-SCC active layer. However, the crosslinking network structure formed in the AETH-TMC active layer could restrict the swelling effect of the ethanol-water feed solution, which would limit the increase in the free volume of AETH-TMC active layer. Thus, a higher water concentration in the permeate and a lower permeation rate for a $90 \mathrm{wt} \%$ ethanol aqueous solution through the AETH-TMC/mPAN thin-film composite membrane were obtained.

The above-mentioned results for water contact angle and PAS experiments can be summed up as follows. TMC in comparison to SCC in the poly(thiol ester amide) backbone caused a decrease in both the hydrophilicity and the free volume of the poly(thiol ester amide) active layer. For the pervaporation separation, the decreasing hydrophilicity causes an increase in the affinity between the poly(thiol ester amide) active layer and ethanol molecules, especially at higher ethanol content of the feed solution. In other words, an increase in the swelling effect of the ethanol aqueous solution has a tendency to increase the free volume. However, crosslinking structure restricts the increase in the free volume resulting from the swelling effect of the ethanol aqueous solution. This makes water molecules enter the membrane more easily than ethanol. In this study, the crosslinking effect during interfacial polymerization played an important role in the poly(thiol ester amide)/mPAN thin-film composite membrane for pervaporation separation.

\subsection{Comparison of ethanol aqueous solution separation performance}

To improve the pervaporation performance in separating ethanol aqueous solutions, many studies on membranes prepared by means of different fabrication or modification methods have been investigated. It is interesting to compare the pervaporation performance in separating ethanol aqueous solutions in this study with that in the literature [15,23-27], as shown in Table 5. PERVAP ${ }^{\circledR}$ 2201 commercial membrane by Sulzer Chemtech and several membranes prepared by different fabrication or modification methods were selected for comparison with AETH-TMC/mPAN in this study. The AETH-TMC/mPAN composite membrane was found to exhibit superior performance than the membranes in the literature. Therefore, the poly(thiol ester amide) thin-film composite membranes could be a candidate for ethanol dehydration by pervaporation.

\section{Conclusion}

The poly(thiol ester amide) thin-film composite membranes were successfully prepared by the interfacial polymerization of AETH and various acyl chlorides, in relation to evaluating the acyl chloride chemical structure effect on the pervaporation performance. In summing up the above-mentioned experimental results, the following several conclusions were obtained:

1. In the ethanol aqueous solution dehydration, the AETH-TMC/ mPAN composite membrane showed a lower permeation rate and a higher water concentration in the permeate than the AETH-SCC/mPAN. A permeation rate of $1586 \mathrm{~g} /\left(\mathrm{m}^{2} \mathrm{~h}\right)$ and a water concentration in the permeate higher than $99 \mathrm{wt} \%$ were obtained with AETH-TMC/mPAN composite membrane in the dehydration of a $90 \mathrm{wt} \%$ ethanol aqueous solution at $25^{\circ} \mathrm{C}$.

2. The $S$ parameters (free volume) of AETH-TMC active layer were lower than those of the AETH-SCC active layer. Aromatic TMC having three functional groups in comparison to aliphatic SCC with two functional groups in the poly(thiol ester amide) backbone caused the free volume to decrease, resulting from the crosslinking structure in AETH-TMC active layer.

Table 5

Comparison of pervaporation performance for ethanol aqueous solution in this study and in the literature [15,23-27]

\begin{tabular}{|c|c|c|c|c|c|}
\hline Membranes & Ethanol concentration in feed (wt\%) & Operation temperature $\left({ }^{\circ} \mathrm{C}\right)$ & Permeation rate $\left(\mathrm{kg} /\left(\mathrm{m}^{2} \mathrm{~h}\right)\right)$ & Separation factor & Ref. \\
\hline AETH-TMC/mPAN & 90 & 25 & 1.60 & 1130 & This study \\
\hline PERVAP $^{\circledR} 2201^{\mathrm{a}}$ & 90 & 60 & 0.10 & 120 & [23] \\
\hline HXDA-BMTC ${ }^{b}$ & 90 & 40 & 1.70 & 240 & {$[15]$} \\
\hline \multirow[t]{2}{*}{ CS-GA-MAc } & 90 & 50 & 0.24 & 991 & \multirow{2}{*}{ [24] } \\
\hline & 90 & 60 & 0.30 & 634 & \\
\hline CSHM-10d & 85 & 50 & 0.89 & 597 & [25] \\
\hline $\mathrm{PE}-\mathrm{g}-\mathrm{AA}^{-} \mathrm{Na}^{+e}$ & 94 & 70 & 0.38 & 376 & [26] \\
\hline Sulfonated PSF & 90 & 25 & 0.70 & 620 & [27] \\
\hline
\end{tabular}

\footnotetext{
a Membrane of Sulzer Chemtech.

b Imidization temperature: $150^{\circ} \mathrm{C}$.

c Chitosan-glutaraldehyde-maleic anhydride.

d Chitosan-silica hybrid membrane containing $10 \mathrm{wt} \%$ 3-aminopropyl-triethoxysilane (APTEOS).

e Polyacrylate-grafted polyethylene membrane loaded with $\mathrm{Na}^{+}$.

${ }^{f}$ Degree of substitution ( $\mathrm{SO}_{3} \mathrm{H} / \mathrm{PSF}$ unit): 2.0.
} 
3. The solution effect dominated the pervaporation separation behavior of the poly(thiol ester amide) thin-film composite membrane as TMC substituting for SCC in the poly(thiol ester amide) backbone.

\section{Acknowledgements}

The authors wish to sincerely thank the Ministry of Economic Affairs, Ministry of Education Affair, and the National Science Council of Taiwan for financially supporting this work.

\section{References}

[1] T. Uragami, S. Yanagisawa, T. Miyata, Water/ethanol selectivity of new organic-inorganic hybrid membranes fabricated from poly(vinyl alcohol) and an oligosilane, Macromol. Chem. Phys. 208 (2007) 756.

[2] Y. Akihiro, R.K. Tyagi, A. Fouda, T. Matsuura, Effect of evaporation time on the pervaporation characteristics through homogeneous aromatic polyamide membranes. II. Pervaporation performances for ethanol/water mixture, J. Appl. Polym. Sci. 60 (1996) 743.

[3] W.H. Chan, C.F. Ng, S.Y. Lam-leung, X. He, O.C. Cheung, Pervaporation of aqueous ethanol solution through poly(amidesulfonamide)s (PASAs) membranes, Polymer 39 (1998) 2461.

[4] X. He, W.H. Chan, C.F. Ng, Water-alcohol separation by pervaporation through zeolite-modified poly(amidesulfonamide), J. Appl. Polym. Sci. 82 (2001) 1323

[5] S.-Y. Kwak, S.G. Jung, Y.S. Yoon, D.W. Ihm, Details of surface features in aromatic polyamide reverse osmosis membranes characterized by scanning electron and atomic force microscopy, J. Polym. Sci. Part B: Polym. Phys. 37 (1999) 1429.

[6] C.K. Kim, J.H. Kim, I.J. Roh, J.J. Kim, The changes of membrane performance with polyamide molecular structure in the reverse osmosis process, J. Membr. Sci. 165 (2000) 189.

[7] A.P. Rao, S.V. Joshi, J.J. Trivedi, C.V. Devmurari, V.J. Shah, Structure-performance correlation of polyamide thin film composite membranes: effect of coating conditions on film formation, J. Membr. Sci. 211 (2003) 13.

[8] J.S. Louie, I. Pinnau, I. Ciobanu, K.P. Ishida, A. Ng, M. Reinhard, Effects of polyether-polyamide block copolymer coating on performance and fouling of reverse osmosis membranes, J. Membr. Sci. 280 (2006) 762.

[9] L. Li, S. Zhang, X. Zhang, G. Zheng, Polyamide thin film composite membranes prepared from 3,4',5-biphenyl triacyl chloride, 3,3',5,5'-biphenyl tetraacyl chloride and $m$-phenylenediamine, J. Membr. Sci. 289 (2007) 258.

[10] N.-W. Oh, J. Jegal, K.-H. Lee, Preparation and characterization of nanofiltration composite membranes using polyacrylonitrile (PAN). II. Preparation and characterization of polyamide composite membranes, J. Appl. Polym. Sci. 80 (2001) 2729.

[11] S.H. Chen, D.J. Chang, R.M. Liou, C.S. Hsu, S.S. Lin, Preparation and separation properties of polyamide nanofiltration membrane, J. Appl. Polym. Sci. 83 (2002) 1112.
[12] A.W. Mohammad, N. Hilal, M.N.A. Seman, Interfacially polymerized nanofiltration membranes: atomic force microscopy and salt rejection studies, J. Appl. Polym. Sci. 96 (2005) 605.

[13] S. Verissimo, K.-V. Peinemann, J. Bordado, Influence of the diamine structure on the nanofiltration performance, surface morphology and surface charge of the composite polyamide membranes, J. Membr. Sci. 279 (2006) 266.

[14] F. Yang, S. Zhang, D. Yang, X. Jian, Preparation and characterization of polypiperazine amide/PPESK hollow fiber composite nanofiltration membrane, J. Membr. Sci. 301 (2007) 85.

[15] J.-H. Kim, K.-H. Lee, S.Y. Kim, Pervaporation separation of water from ethanol through polyimide composite membranes, J. Membr. Sci. 169 (2000) 81.

[16] S.H. Huang, C.L. Li, C.C. Hu, H.A. Tsai, K.R. Lee, J.Y. Lai, Polyamide thin-film composite membranes prepared by interfacial polymerization for pervaporation separation, Desalination 200 (2006) 387.

[17] C.L. Li, S.H. Huang, D.J. Liaw, K.R. Lee, J.Y. Lai, Interfacial polymerized thin-film composite membranes for pervaporation separation of aqueous isopropanol solution, Sep. Purif. Technol. 62 (2008) 696.

[18] H.M. Chen, W.S. Hung, C.H. Lo, S.H. Huang, M.L. Cheng, G. Liu, K.R. Lee, J.Y. Lai, Y.M. Sun, C.C. Hu, R. Suzuki, T. Ohdaira, N. Oshima, Y.C. Jean, Free-volume depth profile of polymeric membranes studied by positron annihilation spectroscopy: layer structure from interfacial polymerization, Macromolecules 40 (2007) 7542.

[19] S.H. Huang, C.J. Hsu, D.J. Liaw, C.C. Hu, K.R. Lee, J.Y. Lai, Effect of chemical structures of amines on physicochemical properties of active layers and dehydration of isopropanol through interfacially polymerized thin film composite membranes, J. Membr. Sci. 307 (2008) 73.

[20] M.Y. Teng, K.R. Lee, S.C. Fan, D.J. Liaw, J. Huang, J.Y. Lai, Development of aromatic polyamide membranes for pervaporation and vapor permeation, J. Membr. Sci. 164 (2000) 241

[21] K.R. Lee, J.Y. Lai, Pervaporation of aqueous alcohol mixtures through a membrane prepared by grafting of polar monomer onto Nylon 4, J. Appl. Polym. Sci. 57 (1995) 961.

[22] Y.C. Jean, P.E. Mallon, D.M. Schrader, Principles and Applications of Positron \& Positronium Chemistry, World Scientific Publishing Co. Pte. Ltd., Singapore, 2003.

[23] D. Van Baelen, B. Van der Bruggen, K. Van den Dungen, J. Degreve, C. Vandecasteele, Pervaporation of water-alcohol mixtures and acetic acid-water mixtures, Chem. Eng. Sci. 60 (2005) 1583.

[24] W. Zhang, G. Li, Y. Fang, X. Wang, Maleic anhydride surface-modification of crosslinked chitosan membrane and its pervaporation performance, J. Membr. Sci. 295 (2007) 130.

[25] J.H. Chen, Q.L. Liu, X.H. Zhang, Q.G. Zhang, Pervaporation and characterization of chitosan membranes cross-linked by 3-aminopropyltriethoxysilane, J. Membr. Sci. 292 (2007) 125

[26] Z.H. Ping, Q.T. Nguyen, S.M. Chen, Y.D. Ding, Pervaporation of water-ethanol mixtures with polyacrylate-grafted polyethylene (PE-g-AA) membranes. Physico-chemical analysis of the transport mechanism, J. Membr. Sci. 195 (2002) 23.

[27] S.H. Chen, K.C. Yu, S.S. Lin, D.J. Chang, R.M. Liou, Pervaporation separation of water/ethanol mixture by sulfonated polysulfone membrane, J. Membr. Sci. 183 (2001) 29. 